\section{La teleodontología como alternativa de atención durante la pandemia por COVID-19}

\section{Teledentistry as an alternative for dental care during the pandemic by COVID-19}

\section{Resumen}

La teleodontología surgió como una alternativa para continuar la atención odontológica durante la pandemia por el COVID-19 debido a la restricción de las consultas presenciales a, solamente, la atención de urgencias y emergencias. Esta nota científica ofrece una visión de las ventajas, desventajas y recomendaciones para la aplicación de la teleodontología en la práctica profesional.

Palabras clave: Salud Bucal; Telemedicina; Odontología; Infecciones por coronavirus (fuente: DeCS BIREME).

\begin{abstract}
Teledentistry emerged as an alternative to continue dental care during the COVID-19 pandemic due to the restriction of consultations to urgent care and dental emergencies. This scientific note offers an overview of the advantages, disadvantages, and recommendations for the application of teledentistry in professional practice.
\end{abstract}

Keywords: Oral Health; Telemedicine; Dentistry; Coronavirus Infections (source: MeSH NLM).
Nota Científica
Grace Revelo 1,a

${ }^{1}$ Universidad Central del Ecuador, Facultad de Odontología, Quito, Ecuador.

${ }^{a}$ Especialista en Estomatología Pediátrica. Especialista en Ortodoncia y Ortopedia Maxilar.

\section{Correspondencia:}

Grace Revelo: gracerevelo@hotmail.com

Quito 170129, Equador

ORCID: 0000-0002-6448-0440

\section{Editor:}

Guido Artemio Marañón Vásquez

Universidade Federal do Rio de Janeiro, Brasil.

Conflicto de intereses: la autora declara no tener conflictos de interés.

Fuente de financiamiento: fondos propios de la autora.

Recibido: 16/01/21

Aceptado: 24/03/21

Publicado: 01/07/21 


\section{Introducción}

La pandemia por COVID-19 representa una emergencia sanitaria y una crisis de salud pública en la mayoría de los países del mundo. Debido a su propagación rápida se incluyeron medidas restrictivas a los proveedores de servicios de odontología quienes tuvieron que postergar la atención electiva, excepto las emergencias y urgencias odontológicas por la posibilidad de contagio al paciente y el equipo de salud ${ }^{1}$. La salud bucodental se considera un pilar fundamental para gozar de una buena salud y calidad de vida, por lo tanto, la odontología es un complemento importante del sistema de atención en salud que se ha visto comprometida durante la actual pandemia de COVID-19. La teleodontología no sustituye la consulta presencial, sin embargo, ha permitido apoyar el sistema de salud durante la pandemia ${ }^{2-4}$.

El término telemedicina se refiere al empleo de la información electrónica y las tecnologías de la computación para proveer servicios de salud entre participantes que están separados. La telemedicina es una forma eficaz de mejorar la calidad de la atención y disminuir el costo de la atención médica a través de un mejor uso de los recursos y el tiempo del profesional de la salud ${ }^{5}$. La teleodontología se propuso en 1994 como un proyecto para los militares en Estados Unidos con la finalidad de mejorar el acceso del paciente al cuidado dental ${ }^{6}$. Una revisión sistemática sobre los beneficios de la teleodontología encontró que la teleodontología tiene un gran potencial para influir en la toma de decisiones respecto al cuidado de la salud y que la teleconsulta puede ayudar a reducir costos en comparación con la consulta normal, sin embargo, la evidencia no es concluyente ${ }^{7}$.

Antes de la pandemia, la teleodontología se permitía en algunos estados y países, sin embargo, con el COVID-19 se extendió su uso al considerarla como una herramienta útil para continuar con la práctica odontológica remota ${ }^{8}$. Su utilización puede beneficiar algunas situaciones clínicas y permite mejorar la salud oral en los niños de las escuelas ${ }^{9,10}$. El propósito de este artículo es ofrecer una visión sobre el marco legal, las ventajas, desventajas y recomendaciones para la aplicación de la teleodontología en la práctica profesional.

\section{Marco legal de la teleodontología en Latinoamérica}

Hasta la fecha, solamente algunos países han emitido regulaciones para la telesalud y la teleodontología.

Para la regulación de la Telesalud en Argentina se dispuso la Resolución 282 de 2020 de la SuperSalud; en Bolivia se cuenta con la Resolución 200 del 2015; en Chile la Resolución 2042020 MinSalud, en Colombia la Resolución 2654 de 2019; en Ecuador el Plan Nacional de Telesalud de marzo 2010; en México el No. Proyecto de Ley de TeleSalud del 2015; en Paraguay la Ley 5.842 de 2015; en Perú la Norma Técnica NTS No 067-MINSA/DGSP-V01, en Uruguay la Ley 19+.869 de 2020 y en Venezuela el No. Anteproyecto Ley de Telesalud del $2015^{11}$.

Para la Teleodontología en el Perú, la Directiva Sanitaria $100 \mathrm{MINSA} / 2020 / \mathrm{DGIESP}$, en el anexo 3, dispone el triaje para determinar el tipo de atención estomatológica donde en los casos que no presentan dolor, no hubo trauma dental o no existe sangrado posterior a una extracción se puede realizar la atención vía telefónica o virtual ${ }^{12}$. En Chile se cuenta con la Res 2020 Minsalud; en Colombia con los Lineamientos Salud Oral en Urgencias en Covid-19 pandemia mayo 2020 y para países como Argentina, Bolivia, Ecuador, Paraguay y Uruguay no existe una regulación referente a la teleodontología, sin embargo, la regla general de TeleSalud no es excluyente a Odontología ${ }^{11}$.

\section{Práctica de la teleodontología durante la pandemia}

La teleodontología ha permitido brindar consultas odontológicas en el marco de la pandemia del COVID-19 y constituye un paradigma emergente para la atención odontológica de los países desarrollados y en vías de desarrollo, evidenciando la necesidad de rediseñar el cuidado de la salud bucal ${ }^{13,14}$.

La teleodontología comprende las siguientes subunidades: la teleorientación, que permite al profesional detectar, guiar y referir al paciente para una consulta cara a cara; el telemonitoreo, que permite visualizar lesiones bucales para su control y valorar el progreso del tratamiento ${ }^{15}$; el teletriaje, que permite disponer los síntomas del paciente para priorizar quienes requieren atención odontológica evitando viajes innecesarios en zonas con dificultades geográficas y socioeconómicas; y la teleconsulta, donde el paciente se comunica con el especialista en odontología e incluye el intercambio de información entre los profesionales sobre el diagnóstico y terapia del paciente con mayor rapidez y precisión ${ }^{15,16}$.

Respecto a las modalidades, la teleodontología puede ser sincrónica, cuando hay una comunicación audiovisual o telefónica con el paciente en tiempo real por medio del uso de videollamadas de WhatsApp, Skype, Google Duo, Zoom, etc.; y, asincrónica cuando la transmisión de los registros diagnósticos y los datos se dan a través de un sistema electrónico como e-mail o WhatsApp para valorar la condición del paciente o brindar un servicio fuera del tiempo real ${ }^{17}$.

Los odontólogos pueden incorporar las consultas digitales en su portafolio de servicios y las universidades pueden trasladar las rotaciones externas de los estudiantes a la teleodontología ${ }^{15,16}$. Un estudio que indagó a los estudiantes de odontología sobre la impresión de la teleodontología y el nivel de comodidad con el encuentro de teleodontología presentó que inicialmente el 64\% de los estudiantes reportaron algún nivel de incomodidad, sin embargo, posterior al uso de simuladores se disminuyó esta incomodidad y se encontró que los simuladores brindan oportunidades para enseñar y probar prácticas de telesalud durante la pandemia ${ }^{8}$. 


\section{Ventajas de la teleodontología}

Entre las ventajas de la teleodontología se incluyen su velocidad, es accesible desde cualquier dispositivo y desde cualquier lugar, según la herramienta empleada evita la necesidad de la conversación, permite mantener una comunicación en horarios diferentes a los de atención, puede ayudar a resolver la lectura de registros diagnósticos, reduce el tiempo de consulta, es de bajo costo. Se pueden reducir el número de citas del paciente programando la primera cita a través de la teleodontología para tomar los antecedentes y el consentimiento del paciente; y en la segunda cita realizar la visita presencial para el examen, diagnóstico y proponer el tratamiento ${ }^{18,19}$.

Un estudio que exploró la perspectiva del paciente respecto a la atención por teleodontología durante la pandemia por el COVID-19 encontró que el 97\% de los participantes estuvieron satisfechos con la teleodontología y estaban dispuestos a seguir con las consultas virtuales para los seguimientos. El 91\% pudo comprender el uso del sistema de la teleodontología, manteniendo una buena comunicación con sus odontólogos. Los resultados de tomografías, radiografías e histopatología fueron compartidos por las consultas virtuales. Los pacientes manifestaron que pudieron expresarse con claridad y sintieron que podían hablar con el odontólogo como si estuvieran en persona. La mayoría de los participantes que emplearon consultas telefónicas tuvieron una buena comunicación por este medio, no obstante, el 9\% de los pacientes que utilizaron la clínica virtual tuvieron problemas con el micrófono requiriendo el uso del altavoz generándose una pequeńa brecha en la confidencialidad de la información. Entre las ventajas que encontraron los pacientes de la teleodontología estuvieron el ahorro de viajes, tiempo y dinero en estacionamientos ${ }^{18}$.

Los adultos mayores quienes tienen un alto riesgo para la infección por el virus pueden beneficiarse del cuidado odontológico por medio de la teleodontología, brindándoles consejos e información sobre nutrición e instrucciones de higiene bucal. Adicionalmente, se recomienda complementar la asistencia con los procedimientos de odontología mínimamente invasiva como el tratamiento restaurador atraumático ${ }^{20}$.

Respecto a las ventajas de la telemedicina en la práctica odontológica, un estudio comparó dos grupos de pacientes, los que tenían una condición de urgencia y los de seguimiento. Todos los pacientes fueron informados sobre la implementación de las consultas remotas por medio de servicios de mensaje y envío de fotografías. Se recolectaron 418 fotos de 57 pacientes, 34 fueron del grupo con condición de urgencia. En general, se encontró que la telemedicina permitió monitorear a todos los pacientes, reduciendo los costos y limitando el contacto humano contribuyendo a disminuir el riesgo de contagio de COVID-19 ${ }^{21}$.

\section{Desventajas de la teleodontología}

Las desventajas de la teleodontología incluyen que puede ser poco aceptada por los odontólogos por su complejidad, el temor a realizar un diagnóstico inapropiado al limitarse solo a la inspección visual sin poder palpar o auscultar las lesiones, puede generar costos adicionales a la práctica, requiere una buena conexión a internet. Por ello, es necesario que se capacite a los odontólogos y en las universidades se enseñe a los estudiantes a emplear la teleodontología como una alternativa para prevenir y controlar las lesiones ${ }^{16}$. Otras desventajas son las siguientes: puede considerarse invasiva, puede haber errores en tipeo e interpretación de ayudas diagnósticas o prescripciones generándose equivocaciones, la privacidad y la confidencialidad de los datos no pueden garantizarse, podría traer implicaciones legales sin la cobertura de las aseguradoras, las imágenes diagnósticas provistas por el paciente podrían tener una baja resolución que no permitirían su uso y el paciente podría sentir una comunicación inadecuada de sus problemas con el odontólogo por la falta de comunicación cara a cara ${ }^{15,16,19}$.

Una investigación sobre la utilización de la teleodontología para la prescripción de antibióticos y diagnóstico de enfermedades infecciosas en cavidad oral en grupos de odontólogos de WhatsApp, encontró que algunos odontólogos no pudieron diagnosticar las infecciones virales y fúngicas, además, en ocasiones no prescribieron la medicación correcta para el paciente, por ejemplo, se recomendó antibiótico en casos de gingivitis o el antibiótico que no era apropiado para una madre en periodo de lactancia. Se recomiendan cursos de educación continua sobre las manifestaciones de infecciones bucales y contar con protocolos de tratamiento que indiquen en qué casos se realiza la prescripción antimicrobiana ${ }^{22}$.

\section{Recomendaciones para realizar la teleodontología}

Entre las recomendaciones para el uso adecuado de las tecnologías de información se encuentran: establecer con el paciente un tiempo de respuesta para los mensajes, establecer un acuerdo en relación con los días y horarios para responder consultas por este medio, acordar con los pacientes el tipo de consultas que se aceptará, informar al paciente sobre la privacidad de la consulta y si aplica, quién podrá tener acceso a los mensajes, estas comunicaciones deben incluirse en la historia clínica, tener un correo electrónico exclusivo para estas consultas e incluir un texto con los términos y condiciones que rigen estas consultas, confirmar la recepción del correo al paciente y el plazo de respuesta, no reenviar textos ni fotografías del paciente a terceros sin el consentimiento del paciente ${ }^{19}$.

Un estudio realizado sobre los conocimientos y actitudes de los odontólogos hacia la teleodontología indicó que el conocimiento y la práctica de la teleodontología antes de la pandemia del COVID-19 era bajo $(25,75 \%$ y $12,42 \%$ respectivamente), sin embargo, desde el inicio de la pandemia se aumentó el conocimiento y la práctica sobre la teleodontología $(62,72 \%$ y 42,53\% respectivamente), el 59,55\% de los odontólogos podrían considerar practicarla después de la pandemia ${ }^{23}$. Otro estudio 
comparó los conocimientos, actitudes y prácticas entre odontólogos generales, estudiantes de posgrado de odontología, docentes de odontología y consultores con grado de posgrado. Se encontró que los odontólogos generales presentaron mayores conocimientos sobre la teleodontología, mientras que los estudiantes de odontología de posgrado tuvieron menos conocimientos ${ }^{24}$.

La teleodontología ha permitido resolver virtualmente muchos problemas de salud bucal manejando algunas situaciones odontológicas sin que el paciente acuda al servicio de salud, sin embargo, debe tenerse en cuenta que no está libre de errores e involucra cuestiones ético-legales que merecen atención para mejorarla $25,26$. Hasta la fecha, en los programas de odontología de pregrado y posgrado no existe una educación formal sobre teleodontología. La pandemia debe tomarse como una oportunidad para incorporarla en la malla curricular de tal forma que juegue un papel fundamental no solo para situaciones de pandemia sino para que los pacientes y especialistas de zonas remotas puedan beneficiarse de las opiniones y mejorar la atención en salud ${ }^{27}$.

\section{Conclusiones}

La teleodontología ha abierto nuevas posibilidades de comunicación en la relación odontólogo-paciente en la época de pandemia y a futuro brindando soluciones de consulta para situaciones donde no sea posible una relación cara a cara. Para llevarla a cabo, es fundamental capacitar a los odontólogos y los estudiantes de odontología sobre la teleodontología y el empleo responsable y prudente de la información que se obtiene y brinda a través de estas tecnologías. También es necesario contar con protocolos específicos de teleodontología que permitan realizar un triaje de emergencia y que sean objeto de estudio en cuanto a su efectividad y validez. Adicionalmente, el odontólogo debe ofrecer información clara al paciente sobre los requerimientos para realizar la consulta a través de este medio y establecer protocolos específicos para mantener la confidencialidad de los datos del paciente.

\section{Referencias bibliográficas}

1. Sigua-Rodríguez A, Bernal-Pérez JL, Lanata-Flores AG, Sánchez-Romero C, Rodríguez-Chessa J, et al. COVID-19 y la Odontología: una Revisión de las Recomendaciones y Perspectivas para Latinoamérica. Int J Odontostomat. 2020;14(3):299-309. DOI: http://dx.doi. org/10.4067/S0718-381X2020000300299.

2. Organización Mundial de la Salud. Salud bucodental [Internet]. [citado el 7 de marzo de 2021]. Disponible en: https://www.who.int/topics/oral_health/es/

3. Morón Araújo M. La Teleodontología una Herramienta Fundamental en Tiempos de Pandemia y post COVID -19, su Utilidad en las Diferentes Especialidades Odontológicas. Int J Odontostomat. 2021;15(1):4350. DOI: http://dx.doi.org/10.4067/S0718$381 X 2021000100043$.

4. Freitas JA, Carvalho IS, Alves FB, Costa N da S, Carvalho GAO. Biossegurança em procedimentos cirúrgicos odontológicos frente a pandemia do COVID-19. Res
Soc Dev [Internet]. 2021 [citado el 7 de marzo de 2021]; 10(1):e5810111401. Disponible en: https://rsdjournal. org/index.php/rsd/article/view/11401.

5. Institute of Medicine (US) Committee on Evaluating Clinical Applications of Telemedicine. Telemedicine: A Guide to Assessing Telecommunications in Health Care. Field M, editor. Washington (DC): National Academies Press (US); 1996.

6. Rocca MA, Kudryk VL, Pajak JC, Morris T. The evolution of a teledentistry system within the Department of Defense. Proc AMIA Symp. 1999;921-924.

7. Estai M, Kanagasingam Y, Tennant M, Bunt S. A systematic review of the research evidence for the benefits of teledentistry. J Telemed Telecare. 2018;24(3):147-56. DOI: $10.1177 / 1357633 X 16689433$

8. Patel SA, Halpin RM, Olson GW, Franklin A. Global pandemic and the rise of teledentistry. J Dent Educ. 2020;(July):1-2. DOI: 10.1002/jdd.12355.

9. Crawford E, Taylor N. The effective use of an e-dentistry service during the COVID-19 crisis. J Orthod [Internet]. 2020 [citado el 8 de noviembre de 2020]; 47(4). Disponible en: https://pubmed.ncbi.nlm.nih.gov/32854562/

10. Estai M, Kanagasingam Y, Mehdizadeh M, Vignarajan J, Norman R, Huang B, et al. Teledentistry as a novel pathway to improve dental health in school children: a research protocol for a randomised controlled trial. BMC Oral Health [Internet]. 2020 [citado el 30 de noviembre de 2020];20(1):11. Disponible en: https://bmcoralhealth.biomedcentral.com/articles/10.1186/s12903-0190992-1\#citeas

11. Equipo de trabajo multidisciplinario de la Revista de Odontopediatría Latinoamericana. Teleodontología: aplicación a la odontopediatría durante la pandemia COVID-19. Rev odontopediatr latinoam [Internet]. 2020 [citado el 7 de marzo de 2021];10(2):1-21. Disponible en: https://www.revistaodontopediatria.org/ediciones/2020/2/art-3/

12. Perú. Ministerio de Salud. Manejo de la Atención Estomatológica en el contexto de la pandemia por COVID-19 [Internet]. 2020 [citado el 7 de marzo de 2021]. Disponible en: https://cdn.www.gob.pe/uploads/document/ file/716209/DIRECTIVA_SANITARIA_N_100-MINSA-2020-DGIESP.pdf

13. Santana LA, Dos Santos MAL, de Albuquerque HIM, Costa SFDS, Rezende-Silva E, Gercina AC, et al. Teledentistry in Brazil: A viable alternative during covid-19 pandemic. Rev Bras Epidemiol. 2020;23:1-3. DOI: 10.1590/1980-549720200082.

14. Martins MD, Carrard VC, Dos Santos CM, Hugo FN. COVID-19- Are telehealth and tele-education the answers to keep the ball rolling in Dentistry? Oral Dis [Internet]. 2020 [citado el 16 de diciembre del 2020];2(10). Disponible en: https://www.ncbi.nlm.nih. gov/pmc/articles/PMC7361312/

15. Telles-Araujo G de T, Caminha RDG, Kallás MS, Santos PS da S. Teledentistry support in COVID-19 oral care. Clinics. 2020;75:1-2. DOI: 10.6061/clinics/2020/e2030.

16. Ghai S. Teledentistry during COVID-19 pandemic. Diabetes Metab Syndr Clin Res Rev. 2020;14(5):933935. DOI: 10.1016/j.dsx.2020.06.029. 
17. Chopra SS, Sahoo NK. Protocol for teledentistry during COVID-19 in Armed Forces dental establishments. Med J Armed Forces India. 2020;76(3):356-359. DOI: 10.1016/j.mjafi.2020.05.016.

18. Rahman N, Nathwani S, Kandiah T. Teledentistry from a patient perspective during the coronavirus pandemic. Br Dent J. 2020;229(3):1-4. DOI: 10.1038/s41415020-1919-6

19. Krynski L, Goldfarb G. La comunicación con los pacientes mediada por tecnología: WhatsApp, e-mail, portales. El desafío del pediatra en la era digital. Arch Argent Pediatr [Internet]. 2018 [citado el 16 de octubre de 2020];116(4):554-559. Disponible en: http://www. codajic.org/node/3125.

20. León S, Giacaman RA. COVID-19 and Inequities in Oral Health Care for Older People: An Opportunity for Emerging Paradigms. JDR Clin Transl Res. 2020;5(4):290292. DOI: $10.1177 / 2380084420934742$.

21. Giudice A, Barone S, Muraca D, Averta F, Diodati F, Antonelli A, et al. Can teledentistry improve the monitoring of patients during the Covid-19 dissemination? A descriptive pilot study. Int J Environ Res Public Health. 2020;17(10). DOI: 10.3390/ijerph17103399

22. Dar-Odeh N, Babkair H, Alnazzawi A, Abu-Hammad S, Abu-Hammad A, Abu-Hammad O. Utilization of Teledentistry in Antimicrobial Prescribing and Diagnosis of Infectious Diseases during COVID-19 Lockdown. Eur J Dent. 2020 Dec;14(S 01):S20-S26. DOI: 10.1055/s0040-1717159.
23. Plaza-Ruíz SP, Barbosa-Liz DM, Agudelo-Suárez AA. Impact of COVID-19 on the Knowledge and Attitudes of Dentists toward Teledentistry. JDR Clin Trans Res [Internet]. 2021 [citado el 7 de marzo de 2021] Feb 25:2380084421998632. Disponible en: https://pubmed.ncbi.nlm.nih.gov/33632011/.

24. Abbas B, Wajahat M, Saleem Z, Imran E, Sajjad M, Khurshid Z. Role of Teledentistry in COVID-19 Pandemic: A Nationwide Comparative Analysis among Dental Professionals. Eur J Dent [Internet]. 2020 [citado el 7 de marzo de 2021];14(S 01):S116-S122. Disponible en: https://pubmed.ncbi.nlm.nih.gov/33383589/.

25. Deshpande S, Patil D, Dhokar A, Bhanushali P, Katge F. Teledentistry : A Boon Amidst COVID-19 Lockdown - A Narrative Review. International Journal of Telemedicine and Applications [Internet]. 2021[citado el 7 de marzo de 2021];2021. Disponible en: https://www.hindawi.com/journals/ijta/2021/8859746/.

26. Roco-Bazáez, JA. Impacto de la Pandemia por COVID-19 (SARS-CoV 2) en la Educación Odontológica: Scoping Review. Int. J. Odontostomat. 2021;15(1):1013. DOI: http://dx.doi.org/10.4067/S0718381X2021000100010.

27. Chopra SS, Sahoo NK. Pandemic proofing dental education. Med J Armed Forces India [Internet]. 2021 [citado el 7 de marzo de 2021];77:S31-6. Disponible en: https://doi.org/10.1016/j.mjafi.2020.11.025 